\title{
Feasibility of Virtual Research Visits in Fox Trial Finder
}

\author{
E. Ray Dorsey ${ }^{\mathrm{a}, \mathrm{b}, *}$, Joseph D. Wagner ${ }^{\mathrm{c}}$, Michael T. Bulla ${ }^{\mathrm{a}}$, Ashley Rizzieri ${ }^{\mathrm{c}}$, Justin Grischkan ${ }^{\mathrm{e}}$, \\ Meredith A. Achey ${ }^{\mathrm{b}}$, Todd Sherer ${ }^{\mathrm{d}}$, Sohini Chowdhury ${ }^{\mathrm{d}}$, Claire Meunier ${ }^{\mathrm{d}}$, Lily Cappelletti ${ }^{\mathrm{d}}$, \\ Charlotte Rocker ${ }^{\mathrm{d}}$, Irene H. Richard ${ }^{\mathrm{a}}$, Heidi Schwarz ${ }^{\mathrm{a}}$, Gail Kang ${ }^{\mathrm{f}}$, Stacy H. Ahmad", \\ Rachel A. Biemiller ${ }^{\mathrm{a}}$ and Kevin M. Biglan ${ }^{\mathrm{a}, \mathrm{b}}$ \\ ${ }^{a}$ Department of Neurology, University of Rochester Medical Center, Rochester, New York, United States \\ ${ }^{\mathrm{b}}$ CHET, University of Rochester Medical Center, Rochester, New York, United States \\ ${ }^{\mathrm{c}}$ University of Rochester, Rochester, New York, United States \\ ${ }^{\mathrm{d}}$ The Michael J. Fox Foundation For Parkinson's Research, New York, United States \\ ${ }^{\mathrm{e}}$ University of Rochester School of Medicine and Dentistry, Rochester, New York, United States \\ ${ }^{\mathrm{f}}$ University of California Berkeley, Berkeley, California, United States \\ ${ }^{\mathrm{g}}$ Department of Cell Biology and Pathology, Thomas Jefferson University, Philadelphia, PA, United States
}

\begin{abstract}
.
Background: Fox Trial Finder is an online registry for individuals with and without Parkinson disease (PD) interested in participating in PD research. However, distance or disability could prevent such individuals from participating in traditional, clinic-based research at major centers.

Objective: Use videoconferencing to connect participants to specialists to: (1) demonstrate feasibility of virtual research visits within this population (2) collect phenotypic data of the participants, (3) validate self-reported diagnosis, and (4) gauge interest in virtual research visits.

Methods: We solicited volunteers throughout the United States through Fox Trial Finder. Interested individuals with PD provided consent, were given web cameras if needed, completed baseline surveys, and downloaded videoconferencing software remotely. Participants had a test connection and assessment appointment which included the Montreal Cognitive Assessment (MoCA), then a virtual research visit with a neurologist who reviewed their history and assessed their PD using a modified Movement Disorders Society Unified Parkinson's Disease Rating Scale. Neurologists assessed PD diagnosis and symptomatology. Physicians and participants were surveyed about their experience.

Results: Of 204 individuals who consented, 166 (81\%) individuals from 39 states completed all visits. The mean age was 62 and mean disease duration was 8.0 years. Mean MoCA score was 26.5, and mean modified MDS-UPDRS motor score was 22.8 (out of a possible 124). Neurologists judged PD as the most likely diagnosis in $97 \%$ of cases. Overall satisfaction with the visits was $79 \%$ (satisfied or very satisfied) among neurologists and $93 \%$ among participants.

Conclusions: Through virtual research visits, neurologists engaged, characterized, and validated self-reported diagnosis in individuals with PD over a broad geography. This model may facilitate future research participation.
\end{abstract}

Keywords: Parkinson disease, telemedicine

\footnotetext{
${ }^{*}$ Correspondence to: E. Ray Dorsey, CHET, University of Rochester Medical Center, 265 Crittenden, Box CU420694, Rochester, NY 14642, USA. Tel.: +1 585276 6824; E-mail: ray. dorsey@chet.rochester.edu.
}

\section{INTRODUCTION}

Clinical trials are often delayed or fail due to difficulty recruiting research participants [1-5]. The Michael J. Fox Foundation built Fox Trial Finder 
(FTF) [6] to address this challenge in PD by idenftifying individuals with and without the disease who are willing to participate in future research studies. As of March 9, 2015, over 42,500 individuals have created profiles in the online registry that matches volunteers to trials [7].

However, participation in clinical research studies is challenging. Distance, disability, and the need for frequent in-person visits are major barriers to participation in clinical trials [10]. A study in Alzheimer disease found that home visits were the factor most likely to enable greater participation in clinical trials [11]. In PD, such concerns are present among those who participate in clinical trials and may be even more prevalent among those that do not [12].

Virtual research visits could reduce the time and travel burden of participating in research just as virtual care visits do for individuals with PD seeking care. $[13,14]$ We sought to determine whether virtual research visits with volunteers in FTF are feasible by evaluating the proportion of participants who completed visits. Additionally, we gathered additional phenotypic data, validated the self-reported diagnosis of the volunteers, and gauged interest in the model.

\section{MATERIALS AND METHODS}

\section{Recruitment}

FTF participants over 18 years of age who reported a diagnosis of PD and had completed profiles in FTF were solicited through FTF. Participants were informed during the consent process that they would need a high-speed internet connection capable of streaming video. Recruitment was initially designed to reach a random sample of FTF participants in the United States by directly messaging volunteers with information about the trial. We identified groups of 50-100 randomly selected FTF members who met the above criteria to invite via FTF message. However, the initial design of the FTF messaging system required the research team to look through up to hundreds of messages to find a member's reply.

To avoid this time-consuming method, we switched to a site-based trial listing, FTF's intended method of recruitment. Trial information was posted onto the FTF website as a trial. When posted, FTF automatically notifies volunteers whose profiles meet the trial's inclusion criteria, directing these individuals to the trial listing. To control the population of FTF members notified about the trial, the trial team created "sites" in a random selection of U.S. zip codes and suggested that individuals diagnosed with PD within 100 miles participate. Thirty-three such sites were created using zip code and population data from the U.S. Census Bureau's 2010 Census [15]. A subset of 269 zip codes and their populations was randomly generated from the complete 2010 Census database. From this subset, 33 zip codes were selected based on population so that a range of small (500-4999 residents, $n=11$ ), medium (5000-9999 residents, $n=11$ ), and large ( $>10,000$ residents, $n=11)$ populations were included. The site-based method greatly reduced the need to search for messages because interested members would contact researchers via email, phone, or new FTF message. This method closely resembles the standard FTF approach to identifying research participants.

\section{Study procedure}

FTF participants who expressed interest in the study were contacted via phone by a member of the research team (JW, MB, AR) to discuss the study and obtain informed consent. If willing, participants were then emailed a baseline survey that included demographic questions and internet usage information based on Pew Internet Surveys [16-18] and parts Ib and II of the Movement Disorder Society - Unified Parkinson's Disease Rating Scale (MDS-UPDRS) [19]. After completing the surveys, participants were emailed a link to download secure virtual visit software (SBR Health, Cambridge, MA) onto their desktop, laptop, or tablet computer that enables participants to "check in" to an online waiting room and includes videoconferencing software (Vidyo, Hackensack, NJ). If needed, participants were also mailed a web camera (Logitech, Newark, CA). The research team provided any necessary technical support to set up the software and webcam by phone. The research team then scheduled and completed a test connection visit with the participant, which included performing the Montreal Cognitive Assessment (MoCA) [20]. The visuospatial/executive subsection and the naming subsection of this assessment [21] were emailed to the participants to be printed out prior to the visit. The participants completed these tasks by writing on the printed version, then held the paper up to allow the researchers to take a screen capture. Subsequently, the completed paper was scanned or mailed back to the study team to be scored. All other sections of the assessment were scored directly during the visit. This remote method of conducting the MoCA has previously been shown to be fairly reliable in a very small study in PD [21]. 
Finally, participants had a virtual research visit with a neurologist specializing in PD (ERD, IHR, HS, GK, SHA, RB, KMB). The neurologist collected a uniform set of diagnostic data by discussing the participants' PD history and treatment, performing the motor portion of the MDS-UPDRS (excluding rigidity and balance) and estimating their disease stage using the Hoehn and Yahr scale [22]. As part of the history, the neurologist assessed self-reported progression, genetic status, family history, motor and non-motor symptoms, characteristics chosen based on the information collected by the FTF registration form. These responses were later compared with the self-reported data in participants' FTF profiles. Based on these assessments, the neurologist determined whether idiopathic PD was the most likely diagnosis. If it was not, the neurologist instructed the participant to discuss their diagnosis with their local physician. After these visits, the specialist recorded their satisfaction with aspects of the visit, and the participants were sent surveys to do the same. The surveys were based on a case series of telemedicine visits for PD [23] and also included open-ended questions regarding their positive and negative experiences with the virtual visit.

\section{Statistical analysis}

Data on feasibility, clinical assessments and survey results were analyzed descriptively. Cohen's kappa coefficients were calculated to assess the level of agreement between self-reported symptoms of PD (per the participant's FTF profile) and those assessed by the neurologist.

\section{RESULTS}

\section{Participants}

Participants were recruited from Fox Trial Finder using randomized, direct messaging over an eight week period. During this period, recruitment messages were sent to 651 FTF members, of whom 45 (6.9\%) consented to participate in the study. When site-based recruitment was implemented over an eighteen week period, 10,391 FTF members were potentially eligible and received notifications. During this period 159 (1.5\%) additional FTF members consented to participate. Of the 204 total participants, 166 (81.4\%) completed the virtual research visit (Fig. 1). Of the 38 individuals who provided consent but did not complete the virtual research visit, $28(13.7 \%)$ were lost to follow-up, 9 (4.4\%) withdrew for either technical problems $(n=3)$, time requirements $(n=3)$, or unspecified reasons $(n=3)$, and $1(0.5 \%)$ was excluded from participation because the previous diagnosis was not PD.

The participants came from 39 states throughout the United States. As shown in Table 1, 153 (92\%) of the 166 participants who finished both visits were white and $87(52 \%)$ were women. The participants mean age was 62 and average disease duration was 8.0 years. In comparison to the general FTF population with PD in $2014(n=21,397)$, the percentage of women was significantly higher $\left(\chi^{2}=13.5, p<0.001\right)$, the average age was significantly lower $(t=3.27, p<0.005)$, and the average disease duration was higher $(t=5.05$, $p<0.0001)$. The participants were overwhelmingly well educated, more than a third were still employed, and two-thirds saw a PD specialist for their condition. Participants who had previously participated in PD research studies or clinical trials $(n=104)$ reported traveling an average of 69 miles (each way) to participate. Those individuals who did not complete the virtual research visit were more likely to be currently employed (48.4\% vs. $35.3 \%$ ). Relative to the general population, participants in this study were more likely to use the internet at home ( $98.8 \%$ vs. $86 \%)$ [17].

\section{Phenotype}

The 166 individuals in the study all completed a remote MoCA and a remote MDS-UPDRS. The average total MoCA score was 26.5 and average modified MDS-UPDRS part III (motor score) was 22.6. The modified MDS-UPDRS excluded measurements of rigidity and balance and therefore had a maximum score of 116 .

Mean duration of the neurologist assessment visit was 37.0 minutes. Participants' FTF profiles were created a mean of 18.4 months before the virtual research visit. Cohen's kappa coefficients were calculated to compare the neurologists' assessment of the participants' history of PD-related symptoms with those that were self-reported on each participant's FTF profile (Table 2). Symptom reports were in fair agreement $(\kappa>0.2)$ while reports of pursuing genetic testing or neurosurgery were in substantial agreement $(\kappa>0.6)$. This analysis was repeated for the participants who had updated their profiles within 12 months of their research visit $(n=51)$ and $\kappa$ increased in 14 out of 21 categories, suggesting that agreement may increase for more recently updated profiles. Participants reported more symptoms, neurosurgeries, and genetic tests during the virtual research visit than on 


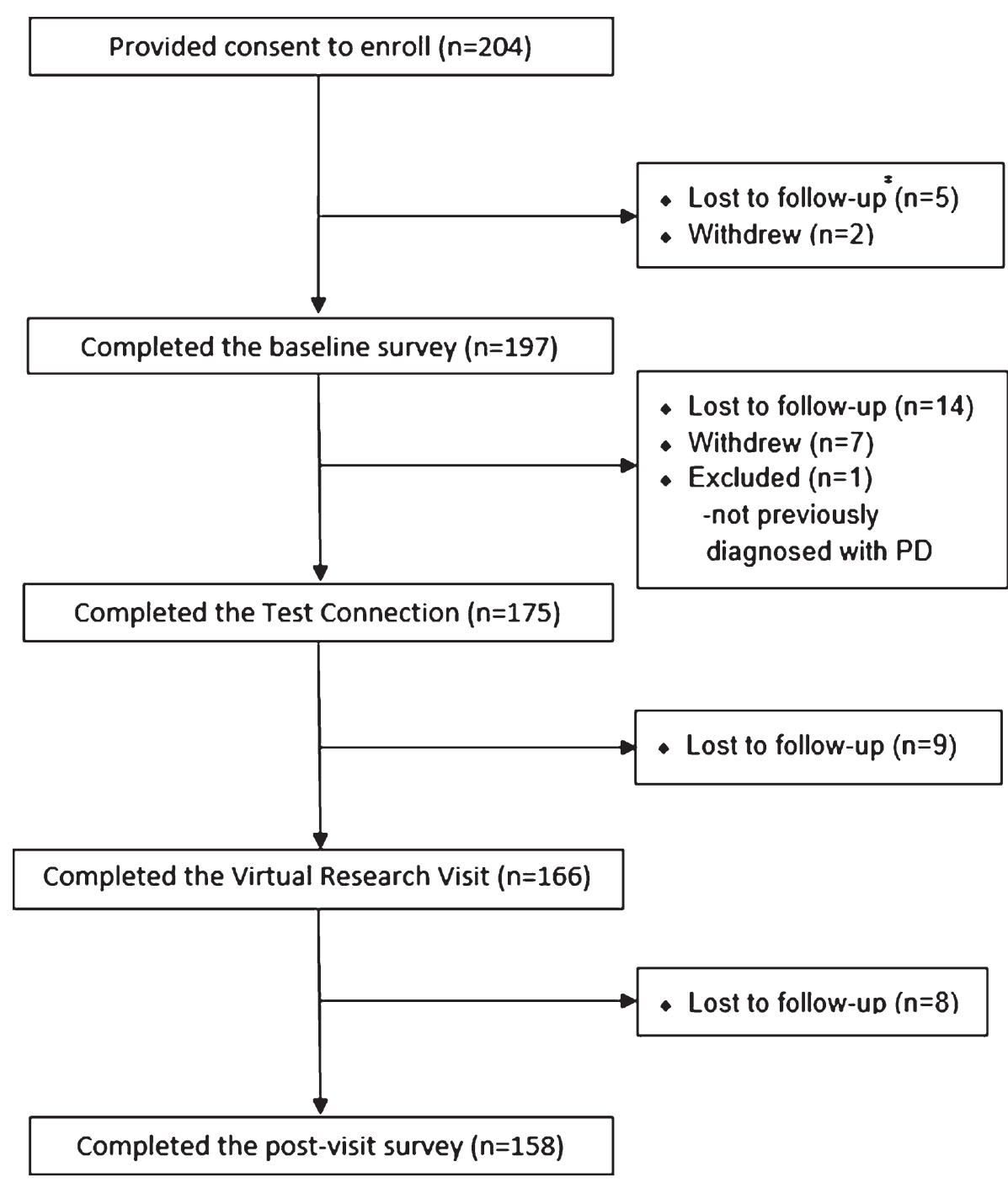

Fig. 1. Progression of Fox Trial Finder participants throughout the study. \#Participants were determined as lost to follow-up after a sustained lack of response to email and phone messages.

their profiles. The neurologist judged PD to be the most likely diagnosis in $97.0 \%$ of cases. The other suggested diagnoses were multiple systems atrophy $(n=2)$, essential tremor $(n=1)$, vascular parkinsonism $(n=1)$, and dystonia $(n=1)$.

\section{Survey}

Neurologists completed $166(100 \%)$ and participants completed $158(95.2 \%)$ of surveys following the 166 virtual research visits. Overall, neurologists were satisfied or very satisfied with $78.8 \%$ of the visits (Fig. 2). Satisfaction with the technical quality of the visit $(77.0 \%)$ and ability to assess the participant remotely $(75.2 \%)$ was more modest. In general, participants expressed higher rates of satisfaction, with $93.0 \%$ satisfied or very satisfied with the overall visit (Fig. 3). In addition, $82.8 \%$ of participants indicated that they would be more able and $87.3 \%$ said they would be more willing to participate in future PD research studies if the studies were conducted virtually.

When asked to describe what they liked about their virtual visits, the participants' responses most often included mentions of the convenience of the visits $(65.0 \%$, "I could be involved in more studies, as it is now I am limited to places real close or have my husband drive me"), their comfort of being home (25.5\%, "the comfort of my own home... I felt more relaxed and felt I communicated better"), or the rapport of the visits $(24.2 \%$, "despite the lack of physical 
Table 1

Characteristics of study participants

\begin{tabular}{|c|c|c|c|}
\hline Demographics & $\begin{array}{c}\text { Completed } \\
\text { virtual research } \\
\text { visit }(n=166)\end{array}$ & $\begin{array}{l}\text { Completed } \\
\text { baseline } \\
\text { survey but not } \\
\text { virtual } \\
\text { research visit } \\
\quad(n=31)\end{array}$ & $\begin{array}{c}\text { Fox Trial } \\
\text { Finder } \\
\text { Population } \\
\text { with PD } \\
(n=21,397)\end{array}$ \\
\hline Age, years, mean (SD) & $61.6(9.5)$ & $60.7(9.2)$ & $64.7(12.2)$ \\
\hline Sex, \% women & 52.5 & 45.9 & 38.5 \\
\hline Race, $\%$ white & 92.2 & 90.3 & 90.9 \\
\hline Education, \% completing high school or more & 98.8 & 96.8 & \\
\hline Education, \% completing 4-years of college or more & 78.9 & 77.4 & \\
\hline Employment, \% currently working & 35.3 & 48.4 & \\
\hline Distance traveled each way for previous studies, miles, mean & 69.1 & 54.3 & \\
\hline Health care provider is PD specialist, $\%$ yes & 67.1 & 64.5 & \\
\hline Duration of condition, $y$, mean (SD) & $8.0(4.5)$ & & $5.8(5.6)$ \\
\hline Technical & $\begin{array}{l}\text { Completed virtual } \\
\text { research visit } \\
(n=166)\end{array}$ & $\begin{array}{l}\text { Completed baseline } \\
\text { survey but not virtual } \\
\text { research visit }(n=31)\end{array}$ & \\
\hline Use internet at home, $\%$ & 98.8 & 100 & \\
\hline with desktop or laptop, \% & 97.0 & 86.7 & \\
\hline have used desktop or laptop for video calling, $\%$ & 42.0 & 50.0 & \\
\hline with smartphone, $\%$ & 50.0 & 56.7 & \\
\hline have used this smartphone for video calling, $\%$ & 53.7 & 29.4 & \\
\hline with tablet, \% & 48.8 & 46.7 & \\
\hline have used this tablet for video calling, $\%$ & 50.0 & 21.4 & \\
\hline Required webcam supplied to participate, $\%$ & 20.5 & 22.6 & \\
\hline $\begin{array}{l}\text { Required }>10 \text { minutes of help to setup video call, } \% \\
\text { Clinical }\end{array}$ & 10.2 & - & \\
\hline Test Connection Visit Overview & Completed Test Connection Visit $(n=175)$ & & \\
\hline Montreal Cognitive Assessment score, mean (SD) & $26.5(2.7)$ & & \\
\hline Virtual Research Visit Overview & Completed Virtual Research Visit $(n=166)$ & & \\
\hline UPDRS- Part I, mean (SD) & $11.4(6.3)$ & & \\
\hline UPDRS- Part II, mean (SD) & $10.7(6.7)$ & & \\
\hline modified UPDRS- Part III, mean (SD) & $22.8(11.4)$ & & \\
\hline Estimated Hoehn and Yahr, mean (SD) & $2.0(0.8)$ & & \\
\hline PD is most likely condition, $\%$ yes & 97.0 & & \\
\hline
\end{tabular}

$\mathrm{SD}=$ Standard deviation.

presence, a warm, reassuring environment was established. To tell the truth, I was very surprised"). When describing what they did not like about the visits, participants' responses most often included technological difficulties or limitations $(40.8 \%$, "interaction was slow because of lag time we had to be careful to not over talk each other"), impersonal interaction (8.9\%, "it is not as personal as being in the same room with a person"), or the limitations of virtual visits ( $7.0 \%$, "the parts of the exam where hands on technique was needed like checking rigidity or balance were missing").

Similarly, when neurologists where asked to describe what they liked about their virtual visit, the neurologists' responses included convenience $(61.7 \%$, "easy to use, saves time compared to clinic"), seeing the participant in their own home (9.9\%, "I could watch her in her daily environment... better assessment of function"), and the rapport ( $8.0 \%$, "interacting with the patient in a more informal environment which allows a bit more candor"). When describing what they did not like about the virtual visit, neurologists' responses included poor visual quality $(28.0 \%$, "fast movements blur"), technical problems (27.5\%, "the call dropped twice, difficulty reconnecting"), and difficulties with the visual field of the camera $(22.8 \%$, "difficulty with camera angles adequately capturing lower extremities, making evaluation challenging").

\section{DISCUSSION}

Rapid advances in telecommunications technology offer the potential to engage research participants in novel, efficient, and powerful ways. In this study, 166 individuals with PD from all over the U.S. were able to undergo remote research assessments that 
Table 2

Reported characteristics of participant's PD history and symptoms

\begin{tabular}{|c|c|c|c|}
\hline PD History & Level of agreement $(\%)$ & Mean squared error (years) & \\
\hline PD is most likely condition, $\%$ yes & 97.0 & - & \\
\hline Year PD symptoms began & 50.1 & 8.13 & \\
\hline Year PD was diagnosed & 86.8 & 0.48 & \\
\hline $\begin{array}{l}\text { Motor symptoms participant } \\
\text { is experiencing }\end{array}$ & Self-reported (\%) & $\begin{array}{c}\text { Response during } \\
\text { neurologist visit }(\%)\end{array}$ & $\begin{array}{c}\text { Cohen's kappa } \\
\text { coefficient }\end{array}$ \\
\hline Falling, poor balance & 21.8 & 35.2 & 0.36 \\
\hline Resting tremor & 69.1 & 75.8 & 0.56 \\
\hline Slowness & 62.4 & 85.5 & 0.26 \\
\hline Small handwriting & 50.9 & 60.6 & 0.42 \\
\hline Trouble walking/shuffled gait & 40.6 & 49.7 & 0.33 \\
\hline \multicolumn{4}{|l|}{ Non-motor symptoms participant is experiencing } \\
\hline Constipation & 39.1 & 52.3 & 0.51 \\
\hline Depression & 29.6 & 41.5 & 0.56 \\
\hline Lightheadedness/orthostatic hypotension & 26.1 & 33.3 & 0.31 \\
\hline Loss of smell & 51.5 & 67.3 & 0.49 \\
\hline Memory loss & 24.2 & 46.1 & 0.37 \\
\hline Pain & 32.7 & 46.1 & 0.20 \\
\hline Sleep disturbances & 62.4 & 72.7 & 0.39 \\
\hline \multicolumn{4}{|l|}{ Medication-induced side effects } \\
\hline Compulsive behavior & 8.5 & 13.3 & 0.44 \\
\hline Dyskinesias & 26.7 & 33.9 & 0.57 \\
\hline Has had deep brain stimulation or other neurosurgery & 4.8 & 8.5 & 0.61 \\
\hline Has a (first degree) family member been diagnosed with PD & 20.7 & 15.5 & 0.77 \\
\hline \multicolumn{4}{|l|}{ Genetic testing } \\
\hline$\%$ that have pursued genetic testing & 40.0 & 52.1 & 0.64 \\
\hline$\%$ that confirmed they have no mutation & 9.7 & 19.4 & 0.43 \\
\hline$\%$ that do not know their results & 25.5 & 27.3 & 0.39 \\
\hline$\%$ with PARK1/PARK4/SNCA or alpha-synuclein & 3.6 & 1.2 & 0.49 \\
\hline$\%$ with PARK2/PARKIN & 0 & 0 & \\
\hline$\%$ with PARK6/PINK1 & 0 & 0 & \\
\hline$\%$ with PARK7/DJ-1 & 0 & 0 & \\
\hline$\%$ with PARK8/LRRK2 or dardarin & 1.2 & 4.2 & 0.43 \\
\hline
\end{tabular}

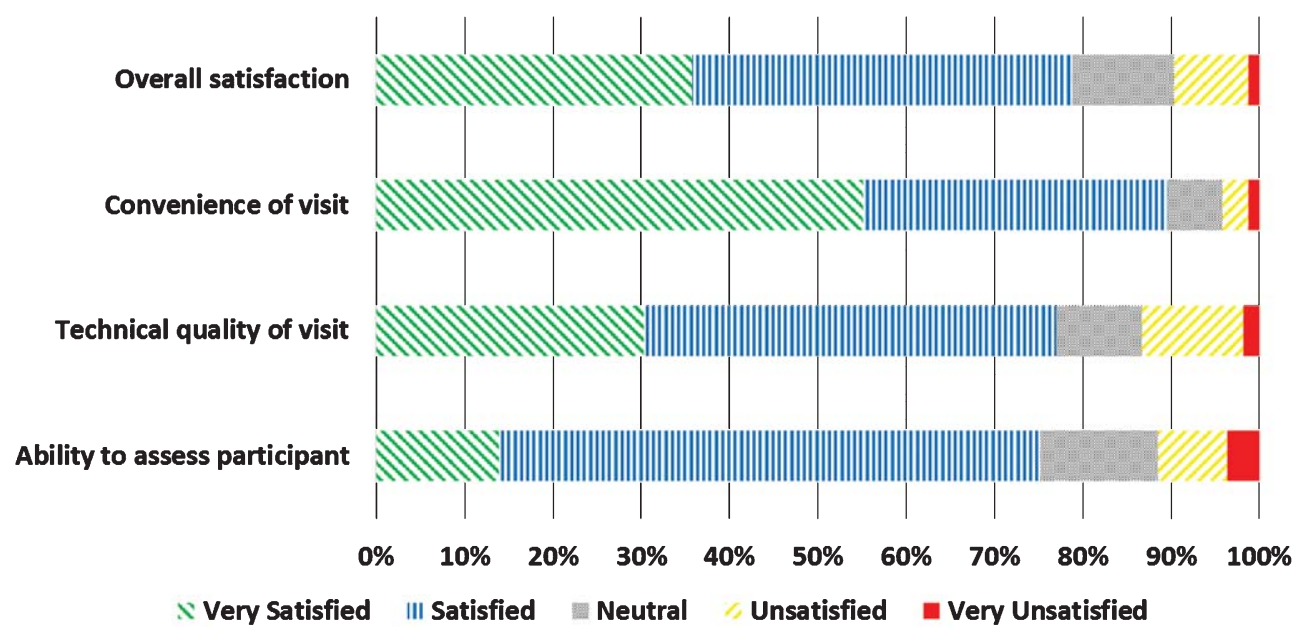

Fig. 2. Satisfaction of physician investigators $(n=166)$ with virtual research visits.

included commonly used clinical outcome measures. Participant feedback suggests that such an approach has considerable promise for engaging individuals in future research endeavors.
In addition, this study validated self-reported diagnosis and added phenotypic data to the largest registry of individuals with PD. Our results suggest that while FTF may be an effective way to identify individuals 


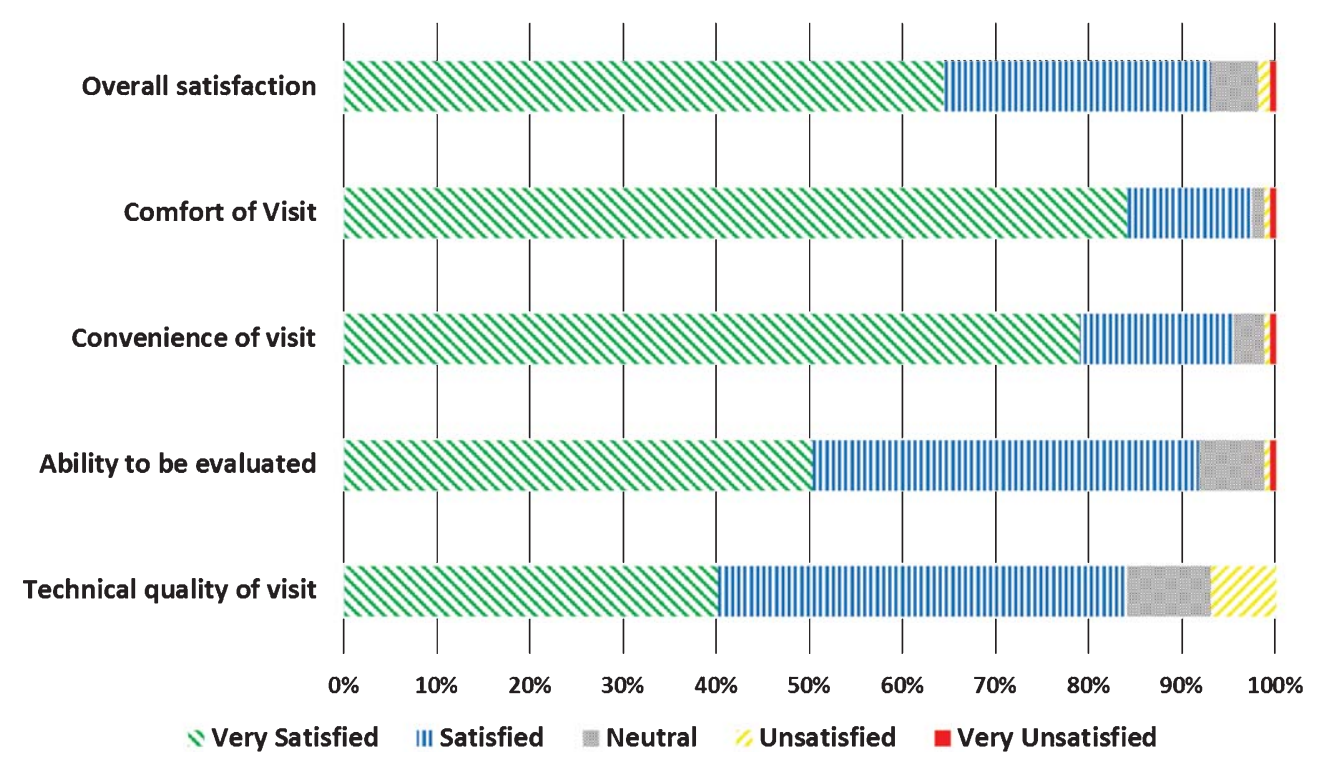

Fig. 3. Satisfaction of FTF participants $(n=158)$ with virtual research visits.

with PD interested in trial participation, the platform may not allow researchers to effectively screen for more in-depth inclusion/exclusion criteria. While agreement with diagnosis was high, the level of agreement of the remote neurologist and the participant on the presence of additional symptoms related to PD was much more modest. In general, the neurologist reported higher rates of motor (e.g., poor balance) and non-motor (e.g., constipation) symptoms than the individual. One likely reason for the discrepancy is the temporal gap between initial completion of the FTF profile by the participant and the assessment by the neurologist. A clinician with expertise in PD may also incite a more sensitive identification of symptoms by the participant during visits.

While the proportion of completed visits and the high satisfaction of both participants and neurologists is promising, this study has several key limitations. First, the population assessed was not a random sample of individuals with PD or even a random sample of FTF participants. On the contrary, the individuals in this study were highly selected, as they were overwhelmingly well-educated, home-Internet users, of whom nearly half had previously made video calls. In fact, the method of recruitment ensured that only individuals with access to internet were included, which excludes a larger portion of low-income and rural individuals. Participants were also required to be functional enough to participate in the virtual assessments as well as operate their computer, or have someone available to help them. This selectivity reflects the current make-up of clinical trial participants fairly accurately [24]. In addition, the increasing ubiquity of the Internet, the rapid rise in its use by older Americans, [25] and the narrowing of the "Digital Divide" [26] generally portend greater (if not accelerated) [27] adoption of the technology. Second, the process by which participants were initially solicited from FTF proved cumbersome, as FTF is not set up to select and contact participants at random. Consequently, trial recruitment was modified to be more closely aligned with FTF's standard methodology, but this limits the generalizability of our results to the larger FTF cohort. Similarly, enrolling in and participating in the study was laborious and involved many steps. Streamlining this process or taking advantage of the larger set-up costs of this method to conduct longitudinal assessments could generate greater value in the future. Third, the technical quality of the visits leaves room for improvement, as identified by both the neurologists and the participants. For example, the quality of the video connection varied with the connection speed available to the participant, which limited the ability to conduct portions of the motor examination. Such limitations will likely decrease as software becomes simpler and broadband connectivity increases. In addition, participants spend much of the video call sitting, such that only the upper trunk and face are visible. Though the whole body and gait are assessed during directed observations, this leaves limited opportunity to assess movements at rest (e.g. rest tremor in hand). Finally, neurologists' satisfaction, while high, did not 
match that of participants. Notably, over $95 \%$ of the visits conducted in this study were by neurologists with very limited or no previous experience in telehealth. With more experience performing telemedicine assessments, the neurologist's ability to evaluate PD and overall satisfaction with these visits may increase. The current alternative to remote assessments is in-person assessments, which places most of the time and travel burden on individuals with PD. Though the principal benefit of telemedicine may be in offering convenience and flexibility to patients, virtual research visits still offer considerable convenience and flexibility to neurologists, many of whom conducted the assessments from their home. Participants and neurologists alike noted that there were advantages to seeing participants in their homes. One participant commented that "the researcher could see my movements in a relaxed normal circumstance," and one neurologist observed that "interacting in the home environment seems to produce more candor."

Despite the limitations of the physical assessment, remote assessments have considerable potential for PD and other movement disorders, as they are primarily assessed visually. While remote assessment of the MDS-UPDRS has not been validated to date, several studies have previously demonstrated high levels of agreement between the motor portion of the UPDRS conducted remotely and in-person [28, 29]. Compared with the complete motor UPDRS, modifying the UPDRS to exclude assessments of rigidity and balance is cross-sectionally and longitudinally reliable [30]. A recent study also suggested that remote assessment of the MoCA in PD and other movement disorders resulted in fair to good reliability compared to an inperson assessment [21]. Other instruments, which rely almost exclusively on ratings by individuals with PD, are even better suited for remote assessments. Indeed, this study builds off the results of the Follow-Up of Persons With Neurologic Diseases (FOUND) study, which demonstrated that remote assessments using mailed questionnaires and telephone interviews to assess PD status was feasible. This resulted in long-term retention of research participants with high levels of agreement between remote and in-person assessments for diagnosis, disease features, and medications [31].

Beyond the mechanics of the remote assessments, their value may be large. In the United States, over $40 \%$ of Medicare (the universal health insurance program for those over 65) beneficiaries with PD do not see a neurologist [32] and thus are unlikely to participate in clinical research studies. The proportion lacking access to neurological care and thus likely research studies is much higher in the vast majority of the world [33-35]. As the disease progresses, disability may even worsen access to care [32]; therefore, patients with the greatest unmet therapeutic needs, have the least access to research. As evidenced by the respondents in this study, remote research visits may increase the ability and willingness of individuals to participate in research, as well as include groups that are generally less likely to participate in PD trials, such as individuals without a PD specialist or women. The National Institute of Neurological Disorders and Stroke also recognizes this potential and called for new means to assess and engage research participants, including investigating "telemedicine to capture long-term outcomes in clinical research cohorts," in its 2014 PD Research Recommendations [36]. Another key advantage of remote assessments is the ability to conduct such assessments from a single "center," which obviates the need for multi-center studies. This removes time and expense for sub-contracts and local institutional review board reviews [37, 38] and has the potential to use centralized raters, which reduces variability in assessments [39-41].

The potential application of virtual research visits in the future is great. Similar to FOUND, they could be used to conduct longitudinal assessments following the conclusion of a clinical trial or as part of an observational study. Since visits are performed from the participant's home, the time and cost of their travel is greatly reduced. A previous study found that a clinical virtual visit can save an individual three hours of time and 100 miles of travel per visit [14]. The principal costs for virtual visits are webcameras $(\$ 25$ each in this study) and video conferencing software ( \$300-500 per month). These costs can be offset by savings from clinic space, which is not needed for virtual assessments. Like audio calls currently, video calls could be used as interim visits in a clinical trial to reduce cost and burden on participants. In addition, they could be used as a screening tool to evaluate potential candidates for clinical trials. Perhaps their greatest value could be in the remote characterization of the natural history of genetic sub-populations of PD or in the enrollment of advanced PD populations in trials aimed at addressing unmet therapeutic needs. This is especially useful when such populations are geographically diverse, residing in nursing homes or not located near traditional research centers [42, 43]. Addressing these opportunities will take time, experience, and improvements in broadband access and connection speeds. This study lays the foundations for the development of these applications, identifying the 
strengths and current challenges for remote evaluation of PD.

\section{ACKNOWLEDGMENTS}

We thank SBR Health and Vidyo for providing their virtual visit and videoconferencing software for use in this study.

\section{AUTHOR ROLES}

Drs. Dorsey and Biglan had full access to all of the data in the study and take responsibility for the integrity of the data and the accuracy of the data analysis.

1. Research project

a. Conception - ER Dorsey, KM Biglan

b. Organization - ER Dorsey, M Bull, KM Biglan

c. Execution - All authors

2. Statistical analysis

a. Design-KM Biglan

b. Execution - J Wagner

c. Analysis and interpretation of the data-All authors

3. Manuscript

a. Wrting of the first draft - ER Dorsey, $\mathrm{J}$ Wagner

b. Review and critique - All authors

\section{FULL FINANCIAL DISCLOSURES OF ALL AUTHORS FOR THE PAST YEAR}

Dr. Ray Dorsey has received compensation for consulting activities from Clintrex, Lundbeck, mc10, Shire, and the National Institute of Neurological Disorders and Stroke, research support from Auspex Pharmaceuticals, Davis Phinney Foundation, Great Lakes Neurotechnologies, Huntington Study Group, Lundbeck, The Michael J. Fox Foundation, Patient-Centered Outcomes Research Institute, Prana Biotechnology, Sage Bionetworks, and stock options from Grand Rounds. He is an uncompensated advisor to SBR Health and Vidyo.

Mr. Wagner, Mr. Bull, Ms. Rizzieri, Mr. Grischkan, and Ms. Achey have no disclosures to report.

Ms. Brooks, Dr. Sherer, Ms. Chowdhury, Ms. Meunier, Ms. Vincent, Ms. Cappelletti, and Ms. Rocker are all employees of The Michael J. Fox Foundation For Parkinson's Research.

Dr. Richard has no disclosures to report.
Dr. Schwarz has no disclosures to report.

Dr. Kang has no disclosures to report.

Dr. Ahmad reports research support from Merz Pharma.

Dr. Biemiller receives research support from NINDS.

Dr. Kevin Biglan received compensation for consulting activities from Lundbeck and Omeros, research support from the Auspex Pharmaceuticals, Davis Phinney Foundation, National Institute of Neurological Disorders and Stroke, The Michael J. Fox Foundation, National Parkinson Foundation, PatientCentered Outcomes Research Institute, Huntington Disease Society of America, and Lundbeck.

\section{FINACIAL DISCLOSURE RELATED TO RESEARCH COVERED IN THIS ARTICLE}

Drs. Dorsey and Biglan have filed for a patent related to neurology and telemedicine. Dr. Dorsey is an unpaid advisor to SBR Health and Vidyo, which provided the software for this study. Dr. Sherer, Ms. Chowdhury, Ms. Meunier, Ms. Cappelletti, and Ms. Rocker are all employees of The Michael J. Fox Foundation For Parkinson's Research.

\section{FUNDING}

This study was funded by The Michael J. Fox Foundation For Parkinson's Research.

\section{REFERENCES}

[1] Lovato LC, Hill K, Hertert S, Hunninghake DB, \& Probstfield JL (1997) Recruitment for controlled clinical trials: Literature summary and annotated bibliography. Control Clin Trials, 18, 328-352.

[2] Haidich AB, \& Ioannidis JP (2001) Patterns of patient enrollment in randomized controlled trials. J Clin Epidemiol, 54, 877-883.

[3] Hunninghake DB, Darby CA, \& Probstfield JL (1987) Recruitment experience in clinical trials: Literature summary and annotated bibliography. Control Clin Trials, 8, 6S-30S.

[4] Easterbrook PJ, \& Matthews DR (1992) Fate of research studies. $J R$ Soc Med, 85, 71-76.

[5] Rocker C, Capelletti L, Marshall C, Meunier CC, Brooks DW, Sherer T, \& Chowdhury S (2015) Use of an online portal to faciilitate clinical trial recruitment: A preliminary analysis of Fox Trial Finder. J Parkinsons Dis, 5, 55-66.

[6] Holden G, Rosenberg G, Barker K, Tuhrim S, \& Brenner B (1993) The recruitment of research participants: A review. Soc Work Health Care, 19, 1-44.

[7] Fox Trial Finder, https://foxtrialfinder.michaeljfox.org, Accessed 10/27-14.

[8] Avis NE, Smith KW, Link CL, Hortobagyi GN, \& Rivera E (2006) Factors associated with participation in breast cancer treatment clinical trials. J Clin Oncol, 24, 1860-1867. 
[9] Mills EJ, Seely D, Rachlis B, Griffith L, Wu P, Wilson K, Ellis P, \& Wright JR (2006) Barriers to participation in clinical trials of cancer: A meta-analysis and systematic review of patient reported factors. Lancet Oncol, 7, 141-148.

[10] Ross S, Grant A, Counsell C, Gillespie W, Russell I, \& Prescott R (1999) Barriers to participation in randomised controlled trials: A systematic review. J Clin Epidemiol, 52, 1143-1156.

[11] Karlawish J, Cary M, Rubright J, \& TenhHave T (2008) How redesigning AD clinical trials might increase study partners' willingness to participate. Neurol, 71, 1883-1888.

[12] Valadas A, Coelho M, Mestre T, Guedes LC, Finisterra M, Noronha A, Rosa MM, Sampaio C, \& Ferreira JJ (2011) What motivates Parkinson's disease patients to enter clinical trials? Parkinson Relat Disord, 17, 667-671.

[13] Achey M, Aldred JL, Aljehani N, Bloem BR, Biglan KM, Chan P, Cubo E, Ray Dorsey E, Goetz CG, Guttman M, Hassan A, Khandhar SM, Mari Z, Spindler M, Tanner CM, van den Haak P, Walker R, \& Wilkinson JR (2014) The past, present, and future of telemedicine for Parkinson's disease. Mov Disord, 29, 871-883.

[14] Dorsey ER, Venkataraman V, Grana MJ, Bull MT, George BP, Boyd CM, Beck CA, Rajan B, Seidmann A, \& Biglan KM (2013) Randomized controlled clinical trial of "virtual house calls" for Parkinson disease. JAMA Neurol, 70, 565-570.

[15] Download 2010 ZIP Code Tabulation Area (ZCTA) Relationship Files - Geography - U.S., United States Census Bureau, https://www.census.gov/geo/maps-data/data/zcta rel_download.html, Accessed March 21, 2014.

[16] Duggan M, \& Smith A (2013) Cell Internet Use 2013. Pew Research Center, http://www.pewinternet.org/2013/ 09/16/cell-internet-use-2013/, Posted September 16, 2013, Accessed Marc, 12, 2015.

[17] Smith A (2014) Older Adults and Technology Use. Pew Research Center, http://www.pewinternet.org/2014/04/03/ older-adults-and-technology-use/, Posted April 3, 2014, Accessed Marc, 12, 2015.

[18] Rainie L, \& Zickuhr K (2010) Video calling and video chat. Pew Research Center, http://www.pewinternet.org/2010/10/ 13/video-calling-and-video-chat/, Posted October 13, 2010, Accessed Marc, 12, 2015.

[19] Goetz CG, Tilley Bc, Shaftman SR, Stebbins GT, Fahn S, Martinez-Martin P, Poewe W, Sampaio C, Stern MB, Dodel R, Dubois B, Holloway R, Jankovic J, Kulisevsky J, Lang AE, Lees A, Leurgans S, LeWitt PA, Nyenhuis D, Olanow CW, Rascol O, Schrag A, Teresi JA, van Hilten JJ, \& LaPelle N (2008) Movement Disorder Society-sponsored revision of the Unified Parkinson's Disease Rating Scale (MDS-UPDRS): Scale presentation and clinimetric testing results. Mov Disord, 23, 2129-2170.

[20] Nasreddine ZS, Phillips NA, Bédirian V, Charbonneau S, Whitehead V, Collin I, Cummings JL, \& Chertkow H (2005) The Montreal Cognitive Assessment, MoCA: A Brief Screening Tool For Mild Cognitive Impairment. J Geriatr Psychiat, 53, 695-699.

[21] Abdolahi A, Bull M, Darwin K, Venkataraman V, Grana M, Dorsey ER, \& Biglan KM (2014) A feasibility study of conducting the Montreal Cognitive Assessment remotely in individuals with movement disorders. Health Informatics $J$ [Epub ahead of print]

[22] Hoehn MM, \& Yahr MD (1967) Parkinsonism: Onset, progression and mortality. Neurology, 17, 427-442.

[23] Venkataraman V, Donohue SJ, Biglan KM, Wicks P, Dorsey ER. Virtual visits for Parkinson disease: A case series. Neurol Clin Pract, 4, 146-152.
[24] Murthy VH, Krumholz HM, \& Gross CP (2004) Participation in cancer clinical trials: Race-, sex-, and age-based disparities. JAMA, 291, 2720-2726.

[25] Madden M (2010) Older Adults and Social Media. Video calling and video chat. Pew Research Center, http://www. pewinternet.org/2010/08/27/older-adults-and-social-media/, Posted August 27, 2010, Accessed Marc, 12, 2015.

[26] Zickuhr K, \& Smith A (2012) Digital Differences. Pew Research Center, http://www.pewinternet.org/2012/04/13/ digital-differences/, Posted April 13, 2012, Accessed Marc, 12, 2015.

[27] Arnol'd VI (1981) Singularity theory : Selected papers, Cambridge University Press, Cambridge Cambridgeshire; New York. (2010), ed. FCC.

[28] Cubo E, Gabriel-Galan JM, Martinez JS, Alcubilla CR, Yang C, Arconada OF, \& Perez NM (2012) Comparison of office-based versus home Web-based clinical assessments for Parkinson's disease. Mov Disord, 27, 308-311.

[29] Dorsey ER, Deuel LM, Voss TS, Finnigan K, George BP, Eason S, Miller D, Reminick JI, Appler A, Polanowicz J, Viti L, Smith S, Joseph A, \& Biglan KM (2010) Increasing access to specialty care: A pilot, randomized controlled trial of telemedicine for Parkinson's disease. Mov Disord, 25, 16521659.

[30] Abdolahi A, Scoglio N, Fau, Killoran A, Dorsey ER, \& Biglan KM (2012) Potential reliability and validity of a modified version of the Unified Parkinson's Disease Rating Scale that could be administered remotely. Parkinsonism Relat Disord, 19, 218-221.

[31] Tanner CM, Meng CC, Ravina B, Lang A, Kurlan R, Marek K, Oakes D, Seibyl J, Flagg E, Gauger L, Guest DD, Goetz CG, Kieburtz K, DiEuliis D, Fahn S, Elliott RA, \& Shoulson I (2014) A practical approach to remote longitudinal follow-up of Parkinson's disease: The FOUND study. Mov Disord, 29, 743-749.

[32] Willis AW, Schootman M, Evanoff BA, Perlmutter JS, \& Racette BA (2011) Neurologist care in Parkinson disease: A utilization, outcomes, and survival study. Neurol, 77, 851-857.

[33] Dorsey ER, Constantinescu R, Thompson JP, Biglan KM, Holloway RG, Kieburtz K, Marshall FJ, Ravina BM, Schifitto G, Siderowf A, \& Tanner CM (2007) Projected number of people with Parkinson disease in the most populous nations, 2005 through 2030. Neurol, 68, 384-386.

[34] Nicoletti A, Sofia V, Bartoloni A, Bartalesi F, Gamboa Barahon H, Giuffrida S, \& Reggio A (2003) Prevalence of Parkinson's disease: A door-to-door survey in rural Bolivia. Parkinsonism Relat Disord, 10, 19-21.

[35] Dorsey ER, \& Willis AW (2013) Caring for the majority. Mov Disord, 28, 261-262.

[36] Trigo-Daporta M, Garcia-Campello M, Perez-Rios M, Santiago-Perez MI, Fernandez-Rodriguez E, Guinarte G, Troncoso A, Pardavila R, Malvar A, \& Pontevedra VPHARpwg (2014) High-risk human papillomavirus in Galicia, Spain: Prevalence and evaluation of the sample representativeness. Scand J Infect Dis, 46, 737-744.

[37] Silberman G, \& Kahn KL (2011) Burdens on research imposed by institutional review boards: The state of the evidence and its implications for regulatory reform. Milbank $Q$, 89, 599-627.

[38] Ravina B, Deuel L, Siderowf A, \& Dorsey ER (2010) Local institutional review board (IRB) review of a multicenter trial: Local costs without local context. Ann Neurol, 67, 258-260.

[39] Kobak KA, Leuchter A, DeBrota D, Engelhardt N, Williams JB, Cook IA, Leon AC, \& Alpert J (2010) Site 
versus centralized raters in a clinical depression trial: Impact on patient selection and placebo response. J Clin Psychopharmacol, 30, 193-197.

[40] Kobak KA, Brown B, Sharp I, Levy-Mack H, Wells K, Ockun F, \& Williams JBW (2009) Sources of unreliability in depression ratings. J Clin Psychopharmacol, 29, 82-85.

[41] Sharp IR, Kobak KA, \& Osman DA (2011) The use of videoconferencing with patients with psychosis: A review of the literature. Ann Gen Psychiatry, 10, 14.
[42] Lesage S, Ibanez P, Lohmann E, Lohmann E, Pollak P, Tison F, Tazir M, Leutenegger A-L, Guimaraes J, Bonnet A-M, Agid Y, Durr A, \& Brice A G2019S LRRK2 mutation in French and North African families with Parkinson's disease. Ann Neurol, 58, 784-787.

[43] Lesage S, Durr A, Tazir M, Lohmann E, Leutenegger A-L, Janin S, Pollak P, \& Brice A (2006) LRRK2 G2019S as a cause of Parkinson's disease in North African Arabs. New Engl J Med, 354, 422-423. 